\title{
3D Printing Endobronchial Models for Surgical Training and Simulation
}

\author{
Zhuo Zhao ${ }^{1}$, Sheng $X u^{2}$, Bradford J. Wood ${ }^{2}$ and Zion Tsz Ho Tse ${ }^{1,3, * \mathbb{D}}$ \\ 1 School of Electrical and Computer Engineering, University of Georgia, Athens, GA 30602, USA; \\ zz67507@uga.edu \\ 2 Center for Interventional Oncology, Radiology and Imaging Sciences, NIH Clinical Center, \\ National Institute of Biomedical Imaging and Bioengineering, \& National Cancer Institute Center for \\ Cancer Research, National Institutes of Health (NIH), Bethesda, MD 20892, USA; xus2@nih.gov (S.X.); \\ BWood@nih.gov (B.J.W.) \\ 3 3T Technologies Limited Liability Company (LLC), GA 30067, USA \\ * Correspondence: ziontse@uga.edu; Tel.: +1-706-542-4189
}

Received: 16 September 2018; Accepted: 14 November 2018; Published: 16 November 2018

\begin{abstract}
Lung cancer is the leading cause of cancer-related deaths. Many methods and devices help acquire more accurate clinical and localization information during lung interventions and may impact the death rate for lung cancer. However, there is a learning curve for operating these tools due to the complex structure of the airway. In this study, we first discuss the creation of a lung phantom model from medical images, which is followed by a comparison of 3D printing in terms of quality and consistency. Two tests were conducted to test the performance of the developed phantom, which was designed for training simulations of the target and ablation processes in endochonchial interventions. The target test was conducted through an electromagnetic tracking catheter with navigation software. An ablation catheter with a recently developed thermochromic ablation gel conducted the ablation test. The results of two tests show that the phantom was very useful for target and ablation simulation. In addition, the thermochromic gel allowed doctors to visualize the ablation zone. Many lung interventions may benefit from custom training or accuracy with the proposed low-cost and patient-specific phantom.
\end{abstract}

Keywords: lung phantom; 3D printing; training and simulation; lung interventions

\section{Introduction}

Lung cancer is the second most common cancer in both men and women [1-6]. More than one million lung cancer cases are diagnosed worldwide each year $[3,7]$. It has the highest death rate among all types of cancers in the United States and worldwide [2,5-9]. According to the American Cancer Society, there were an estimated 222,500 new cases and 155,870 deaths from lung cancer in the United States in 2017 [6]. Early detection and diagnosis as well as an accurate localization during lung intervention may help reduce the death rate for lung cancer [7].

Accurate clinical information can help clinicians choose the optimal treatment for patients, which can improve clinical outcomes [10,11]. An accurate localization of medical devices can significantly improve the accuracy of the diagnosis, which further helps clinicians make the optimal decision via a lung treatment decision tree.

Many methods have been reported for localization in lung interventions. One widely used method is computer-assisted intervention (CAI) due to its advantages of having an increased accuracy, a reduced amount of complications, and a decreased intervention time. Within the field of CAI, image-guided therapy (IGT) has been on the rise for the freehand navigation, guidance, and localization 
of medical devices [12]. Methods for lung interventions using position and orientation include ultrasound (US) imaging, magnetic resonance (MR) imaging, and positron emission tomography (PET)/computed tomography (CT) scanning. Nevertheless, these methods can be imprecise and inaccurate since they cannot provide real-time position and orientation feedback without continually imaging the patient [13]. In order to improve the accuracy of lung interventions, real-time tracking invasive medical tools were developed such as electromagnetic (EM) tracking systems and catheters.

Doctors often train by practicing how to operate these real-time tracking tools in a realistic phantom before using them in a clinical setup with anatomical and physical constraints. These constraints include the intricate structure of lung airways, the complex operation of these devices, and the interpreted spatial relationship between tools and anatomy based on 2D images displayed in the navigation software.

Many studies were conducted regarding the development of lung phantoms. Chang et al. developed a deformable lung phantom for the evaluation of deformable registration [14]. Kairn et al. developed a lung phantom with acrylonitrile butadiene styrene (ABS) [15]. Followill et al. described a lung phantom developed by Radiological Physics Center (RPC) that verifies the accuracy of radiation delivery [16]. There are also some commercially available lung phantoms such as the RANDO lung phantom developed by The Phantom Laboratory (Salem, NY, USA) and an inhomogeneous anthropomorphic lung phantom developed by Computerized Imaging Reference Systems, Inc. (CIRS, Norfolk, VA, USA). All of the previous phantoms focus on lung lobes and none of them have anatomically accurate lung airways in the phantom. These phantoms are, thus, impossible for doctors to use to practice operating real-time tracking invasive medical tools.

Usually, operative resection is the first invasive therapeutic choice for lung malignancies, but there are many factors that limit the application of a surgical approach. For example, only $20 \%$ of all diagnosed lung tumors are resectable and patients with recurrent diseases are not suitable for repeated resections $[17,18]$. Therefore, the radiofrequency $(\mathrm{RF})$ or microwave ablation technique has attracted a great deal of interest as an alternative method for the treatment of lung malignancies. RF ablation is a minimally invasive procedure that uses the heat generated from a medium frequency alternating current to ablate dysfunctional tissues or tumors [19]. The advantages of RF ablation include a reduced procedure-related mortality, shorter recovery periods, preservation of lung function, acceptable two-year survival rates [17], and the ability to combine with IGT. After the position of the tumor is located using real-time tracking invasive medical tools, the RF ablation catheter can then be guided to the tumor for treatment.

In this study, an inexpensive and patient-specific lung airway phantom with RF ablation indicator was hypothesized for pulmonologists to plan, simulate, and practice endobronchial interventions. A rapid-prototype was obtained by using 3D UV resin printing.

\section{Materials and Methods}

Three-dimensional printing technology can fabricate a 3D object from digital designs with no milling or molding. Currently, it has been widely used for rapid prototyping due to its low cost and high repeatability. In this study, we used 3D printing technology to build our lung phantom, which was designed to meet the special requirements set by our pulmonologist: low-cost, patient-specific, and easy visualization of catheter and ablated spots, which are comparable to the human adult size.

\subsection{D Model Design}

In order to build the phantom, a high-quality 3D lung airway model is necessary. A scanned 3D lung airway model with dimensions of $195 \mathrm{~mm} \times 175 \mathrm{~mm} \times 213 \mathrm{~mm}$ (Length $\times$ Width $\times$ Height) was segmented from a real patient, as shown in Figure 1a. However, this model could not be directly used for printing since the inside of the lung airway was completely solid in the scanning model, which is shown in Figure 1b. This means that invasive medical tools or ablation catheters could not be inserted into the phantom. In order to obtain a lung airway phantom with lumen, the scanning 3D model 
needed to be rebuilt. A free STL editor software, Meshmixer (Autodesk Inc., San Rafael, CA, USA), was used for rebuilding purposes. The inspection function was used to repair the scanning model and then the hollow function was applied to build an inner wall of the lung airway. Next, the separate shells function was used, but it only removed a small part of the solid inside of the lung airway due to the model's complexity. The residual part of the solid was removed by first using the place cut function to cut the model into several small parts and then using the separate shells function to remove the solid part. Lastly, the combined function was applied, which recombined all the parts to obtain a useable 3D lung airway model with lumen. This is shown in Figure 1c.

(a)

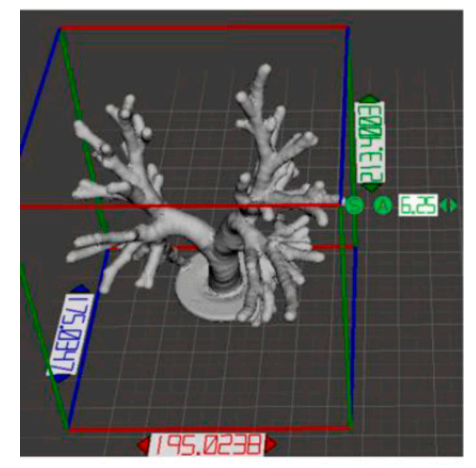

(c)

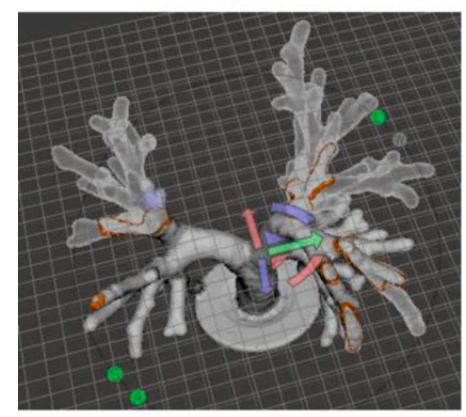

(b)

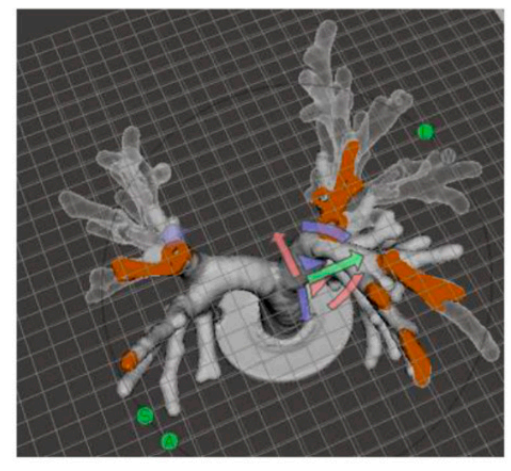

(d)

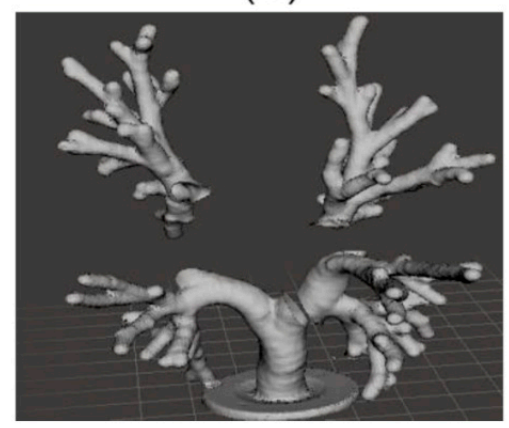

Figure 1. (a) Scanning model from NIH. (b) Inner view of the scanning model. (c) Inner view of the rebuild model. (d) Printed model in the Form 2 printer.

\subsection{D Printing}

There is a variety of 3D printers as well as materials available on the market. We used two printers that were available in our lab to print this model and we then compared the results in order to find a reasonable material as well as an efficient printer. The first printer used was the Lulzbot 3D printer (Aleph Objects, Inc, Loveland, CO, USA) with a print area of $280 \mathrm{~mm} \times 280 \mathrm{~mm} \times 250 \mathrm{~mm}$ (Length $\times$ Width $\times$ Height), which is big enough to print our model. The next printer was the Form $23 \mathrm{D}$ printer (Formlabs, Cambridge, MA, USA) with a build volume of $145 \mathrm{~mm} \times 145 \mathrm{~mm} \times 175 \mathrm{~mm}$ (Length $\times$ Width $\times$ Height), which is small when compared to a realistic lung model. In order to make the phantom closer to the realistic lung airway, the original size of the model must be kept. Therefore, the model used in the Form 2 printer was further processed. The model was then divided into four parts by using the plane cut function in Meshmixer, which has a printable size for Form 2 . This is shown in Figure 1d. Due to the characteristics of the resin, the four printed parts could be connected by placing them under a powerful UV light, which was made by our lab for several minutes. Clear material was used for both printers. 


\subsection{Phantom Performance Test}

\subsubsection{Localization Test}

OncoNav real-time tracking software, which was developed by NIH, was used in the localization test. The CT images of the phantom were acquired first. The OncoNav then read the CT images and reconstructed the phantom, which is shown in Figure 2, in four different views: axial, sagittal, coronal, and 3D. A customized EM tracking catheter was used as a navigation tool. After the registration between the navigation tool and the CT images of the phantom in OncoNav, the real-time position of the catheter in the phantom could be displayed in OncoNav and a target point could be set. The relative position and orientation relationship between the navigation tool and the target point is then displayed on the screen, which allows the tool to be guided in order to reach the target point. The measurement in this test was taken to be the difference between the guided point and the target point. The EM tracking catheter was passed through a hollow tube as the working channel and then retracted from the tube after it reached the target point, which allowed other devices (e.g., forceps) to be deployed at the same target location through the tube.

(a)

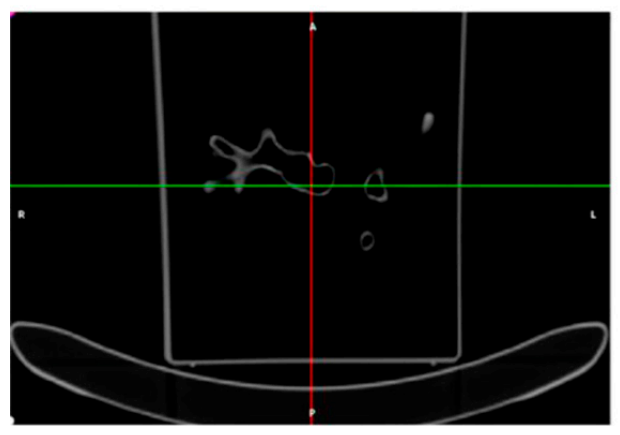

(c)

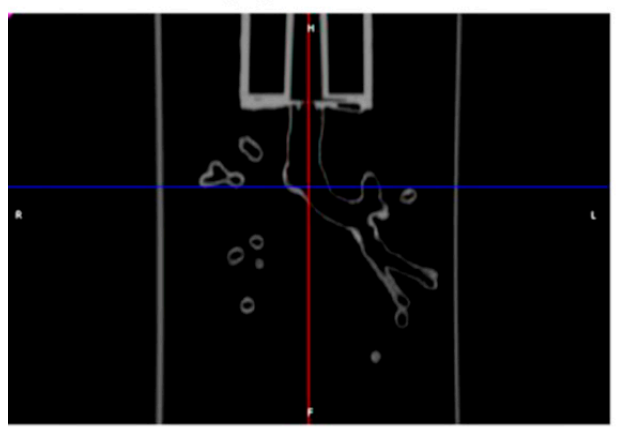

(b)

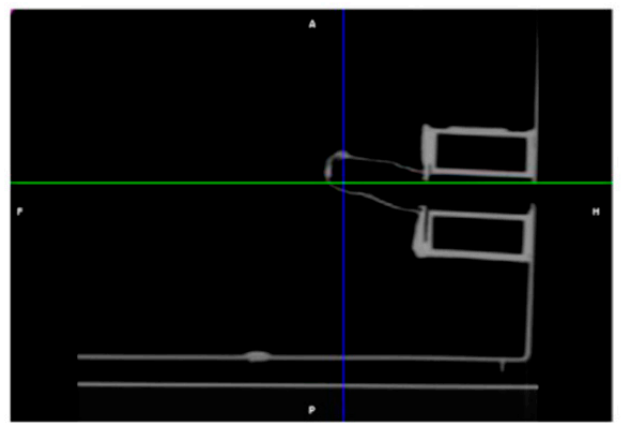

(d)

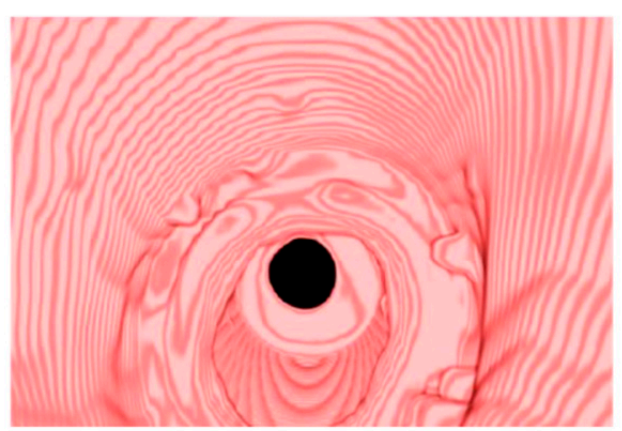

Figure 2. Interface of OncoNav tracking software. (a) Axial view. (b) Sagittal view. (c) Coronal view.

(d) 3D airway reconstruction.

\subsubsection{Ablation Test}

In order to validate ablation, a thermochromic gel was developed as an ablation indicator. The thermochromic gel was made based on the thermochromic ink (ThermometerSite, Glenview, IL, USA) and prepared, as described by Mikhail et al. This thermochromic ink can gradually change color from white to magenta when temperature is between $25^{\circ} \mathrm{C}$ and $75^{\circ} \mathrm{C}$. The colorimetric response of developed gel has been quantified by our team in NIH by fitting the red, greed, and blue (RGB) color channel values and temperature into a nonlinear function [20].

An ablation catheter with a cardiac ablation generator from St. Jude Medical (Saint Paul, MN, USA) was used in the ablation test. During the test, the thermochromic gel was infused into the 
lung airway through the hollow tube after the localization test. Afterward, the ablation catheter was inserted into the phantom through the hollow tube in order to reach the target point. Lastly, the hollow tube was removed carefully and the ablation generator was set up at $70{ }^{\circ} \mathrm{C}$ for $60 \mathrm{~s}$.

\section{Results}

\subsection{Lung Airway Phantom}

The lung airway phantoms printed by the Lulzbot and Form 2 printers are shown in Figure 3a,b, respectively. We compared several characteristics of these two phantoms in Table 1. The phantom from Form 2 was more expensive than the Lulzbot. However, the phantom from Form 2 was higher quality and needed less time to finish printing. The biggest advantage of the phantom from Form 2 was its complete transparency, which is shown in Figure 3. This characteristic was extremely important for the phantom since its purpose was to help physicians gather experience in using medical tools for image-guided lung interventions. The transparent phantom allows the user to visually observe the exact position of the navigation tools. Therefore, the phantom aids the user to build and understand the spatial relationship between tools and anatomy based on 2D images displayed in the navigation software.

Table 1. Comparison of characteristics between the two phantoms.

\begin{tabular}{ccc}
\hline Characteristics & Lulzbot & Form $\mathbf{2}$ \\
\hline Printing Time & $40 \mathrm{~h}$ & $30 \mathrm{~h}$ \\
Amount of material/money & $300 \mathrm{~g} / \$ 15.6$ & $200 \mathrm{~mL} / \$ 29.8$ \\
Resolution & $0.2 \mathrm{~mm}$ & $0.05 \mathrm{~mm}$ \\
Surface & Rough & Smooth \\
Transparency & Opaque & Completely transparent \\
\hline
\end{tabular}

(a)

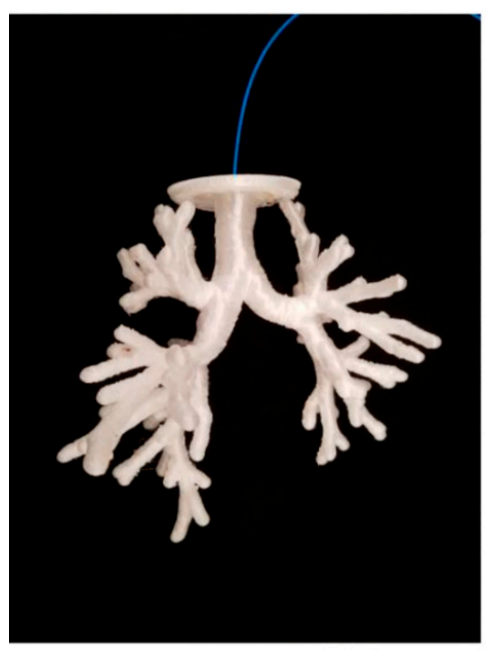

(b)

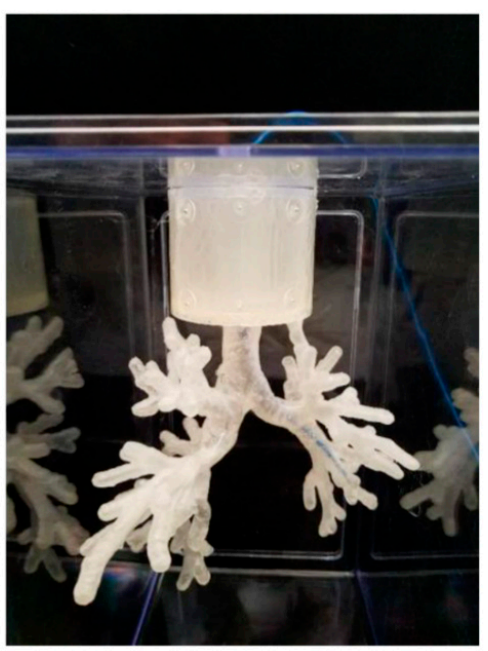

Figure 3. (a) Lung airway phantom from Lulzbot. (b) Lung airway phantom from form 2.

In order to achieve accurate registration between medical tools used for image-guided lung interventions and $\mathrm{CT}$ images of the phantom, a cylinder base with six registration points was created. These registration points had embedded bearing balls (Figure 4a), which appeared as bright spots in the CT images (Figure $4 \mathrm{~b}$ ). The phantom was fixed into a clear acrylic enclosure, which is shown in Figure $3 b$, in order to improve the portability of the phantom as well as to acquire CT images conveniently. 
(a)

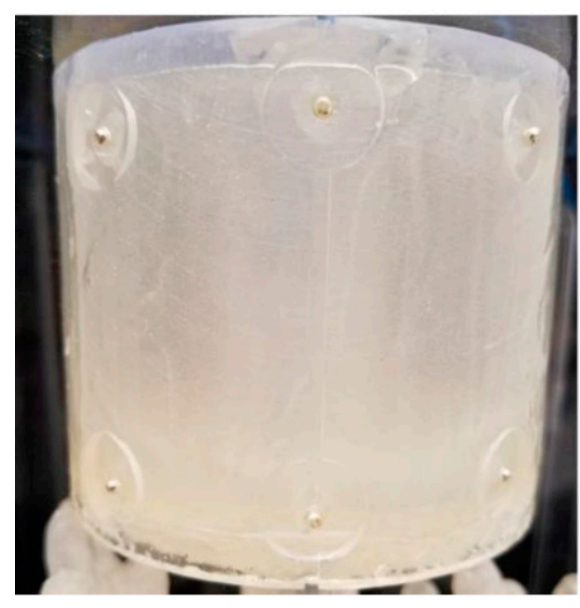

(b)
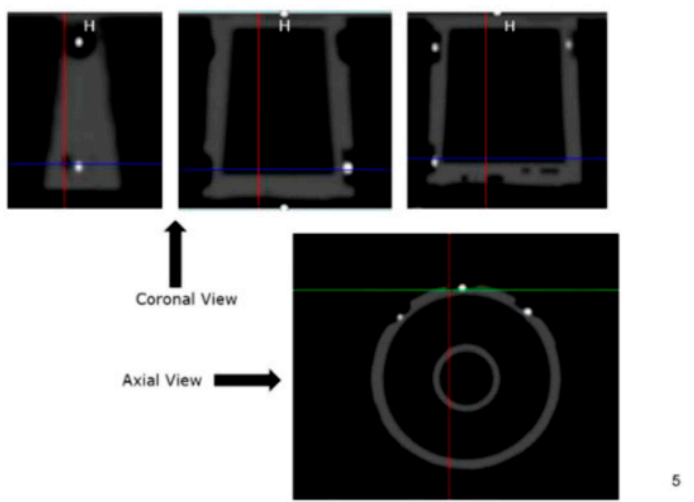

Figure 4. (a) Cylinder base with registration points. (b) Registration points in CT images.

\subsection{Phantom Performance Test}

Ten ends of lung airways were randomly selected with different difficultly levels as target points for the navigation test. The EM tracking catheter was navigated to target points successfully in all trials. The difference between the guided point and the target point had an average value of $1.07 \mathrm{~mm}$ and a standard deviation of $0.58 \mathrm{~mm}$, which is shown in Figure 5. After removing the navigation catheter from the hollow tube, the thermochromic gel and ablation catheter were inserted into the lung airway through this hollow tube and the tube was then carefully removed. The ablation result is shown in Figure 6. After ablation, the indicator changed color from white to magenta. In addition, a color map shown in Figure 7 was created to help visual assessment of temperature change based on the previous work of our team, as described in Reference [20].

\section{Error Distribution}

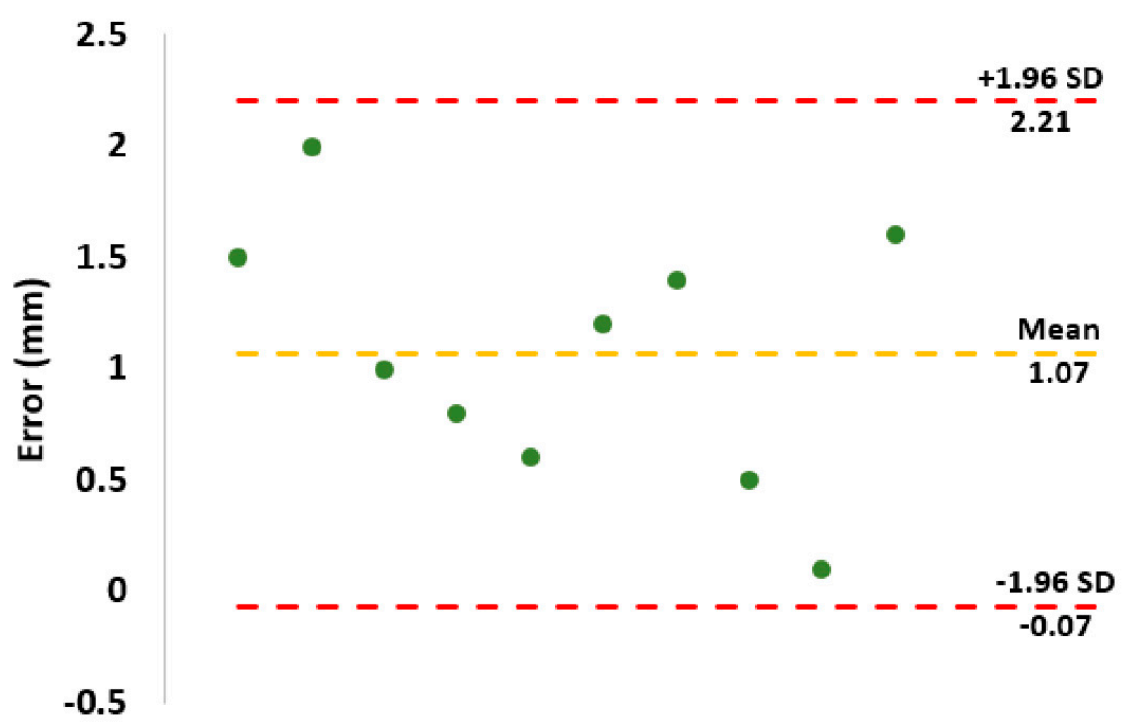

Figure 5. Error distribution of localization test. 
(a)

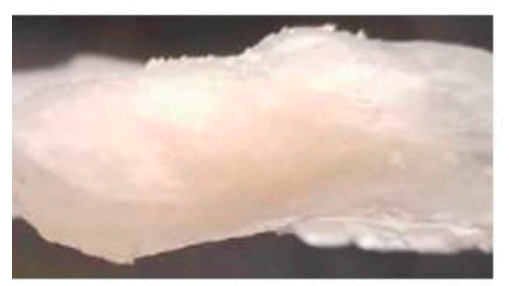

(b)

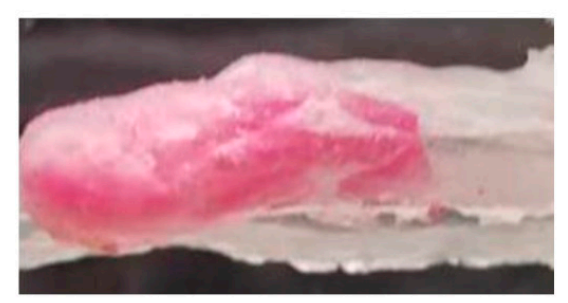

Figure 6. Catheter with an ablation indicator (a) before ablation and (b) after ablation.

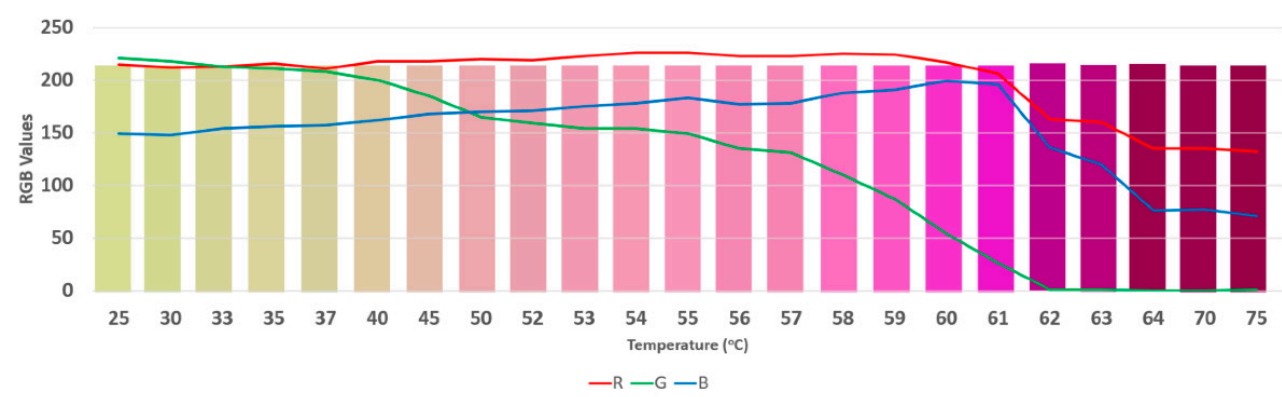

Figure 7. Color map based on the developed thermochromic gel for visual assessment of temperature change.

\section{Discussion}

A 3D printed lung airway phantom was developed in this study and this phantom may prove helpful to train and on which to practice when using medical tools for image-guided lung interventions. In addition, the ablation indicator developed was able to indicate the lung ablation treatment successfully. The whole phantom was inexpensive and the total cost was less than \$100 USD.

However, there are still several aspects that can be improved. First of all, it is not convenient to add the ablation indicator to the target point every time. Ideally, the phantom would be printed from thermochromic material so that the target point would change color after contact with the ablation catheter. However, if we cannot find a clear thermochromic material, we will lose the advantages of the transparent phantom, which makes it difficult for new users to build an understanding of the spatial relationship between tools and anatomy based on 2D images displayed in the navigation software.

Another improvement is based on lung deformation, which is a major issue in lung intervention. Respiratory motion causes lung deformation, which displaces a lung nodule by more than $20 \mathrm{~mm}$ during a respiratory cycle [21]. In the proposed phantom, we did not consider the effect of respiratory motion since the printing material we used is not elastic. Building this phantom with a clear elastic material such as silica gel could allow the effect of respiratory motion in the phantom and result in an increase in time, complexity, and expense due to the cost of labor stemming from the inability to 3D print. Even though the lung airway phantom and ablation indicator we developed are not perfect, they can still be used for general training or practicing at a low cost. In addition, it can be used for testing new lung intervention tools or devices for accuracy or anatomic considerations such as flexibility and radii of curvature.

\section{Conclusions}

In this study, the method of making an inexpensive and patient-specific lung phantom from medical images is first presented. A lung phantom with design specifications from our pulmonologist was then printed using 3D UV resin printing after a comparison of 3D printing in terms of quality and consistency. Next, target and ablation tests were conducted. The results indicated the phantom's ability to be used for training or simulating medical tools in image-guided lung interventions. The developed 
phantom makes it very easy to compare the actual position of a medical tool with the planned position from tracking software during localization, accuracy testing, training, and simulation. In addition, the developed thermochromic gel was very helpful to visualize the ablated volume after the ablation process. The phantom could provide a low-cost way for surgical training and a convenient way for researchers to calibrate and evaluate new medical tools or devices.

Supplementary Materials: The STL files used in this study can be download from: https://www.dropbox.com/ sh/o0jkpr0nywsr4ah/AAABe1L39ynax6Ypb7pZGfxVa?dl=0. It includes the whole lung model and four divided parts for printing.

Author Contributions: Z.T.H.T. conceived and designed the experiments. Z.Z. performed the experiments, analyzed the data, and wrote the paper. S.X. and B.J.W. contributed CT scanning resources and OncoNav software.

Funding: This study was supported in part by the National Institutes of Health (NIH) Bench-to-Bedside Award, the NIH Center for Interventional Oncology Grant, the National Science Foundation (NSF) I-Corps Team Grant (1617340), NSF REU site program 1359095, the UGA-AU Inter-Institutional Seed Funding, the American Society for Quality Dr. Richard J. Schlesinger Grant, the PHS Grant UL1TR000454 from the Clinical and Translational Science Award Program, and the NIH National Center for Advancing Translational Sciences, and the Intramural Research Program of the NIH.

Conflicts of Interest: The authors declare no conflict of interest. The founding sponsors had no role in the design of the study, in the collection, analyses, or interpretation of data, in the writing of the manuscript, or in the decision to publish the results.

\section{References}

1. Siegel, R.L.; Miller, K.D.; Jemal, A. Cancer statistics, 2015. CA Cancer J. Clin. 2015, 65, 5-29. [CrossRef] [PubMed]

2. Uneri, A.; Nithiananthan, S.; Schafer, S.; Otake, Y.; Stayman, J.W.; Kleinszig, G.; Sussman, M.S.; Prince, J.L.; Siewerdsen, J.H. Deformable registration of the inflated and deflated lung in cone-beam CT-guided thoracic surgery: Initial investigation of a combined model-and image-driven approach. Med. Phys. 2013, 40, 017501. [CrossRef] [PubMed]

3. Klein, T.; Traub, J.; Hautmann, H.; Ahmadian, A.; Navab, N. Fiducial-free registration procedure for navigated bronchoscopy. In Medical Image Computing and Computer-Assisted Intervention-MICCAI 2007. MICCAI 2007. Lecture Notes in Computer Science; Ayache, N., Ourselin, S., Maeder, A., Eds.; Springer: Berlin/Heidelberg, Germany, 2007; Volume 4791, pp. 475-482.

4. Xue, Z.; Wong, K.; Wong, S.T. Joint registration and segmentation of serial lung CT images for image-guided lung cancer diagnosis and therapy. Comput. Med. Imaging Graph. 2010, 34, 55-60. [CrossRef] [PubMed]

5. Centers for Disease Control and Prevention (CDC). Lung Cancer, 2017. Available online: https://www.cdc. gov/cancer/lung/ (accessed on 16 September).

6. American Cancer Society. How Common is Lung Cancer? 2017. Available online: https://www.cancer.org/ cancer/non-small-cell-lung-cancer/about/key-statistics.html (accessed on 16 September 2018).

7. He, T.; Xue, Z.; Lu, K.; Valdivia y Alvarado, M.; Wong, K.K.; Xie, W.; Wong, S.T. A minimally invasive multimodality image-guided (MIMIG) system for peripheral lung cancer intervention and diagnosis. Comput. Med. Imaging Graph. 2012, 36, 345-355. [CrossRef] [PubMed]

8. U.S. Food and Drug Administration (FDA). Lung Cancer and Smoking, 2017. Available online: https: //www.fda.gov/tobaccoproducts/publichealtheducation/healthinformation/ucm468635.htm (accessed on 16 September).

9. U.S. National Library of Medicine. Lung Cancer, 2017. Available online: https://medlineplus.gov/ lungcancer.html (accessed on 16 September 2018).

10. Potti, A.; Mukherjee, S.; Petersen, R.; Dressman, H.K.; Bild, A.; Koontz, J.; Kratzke, R.; Watson, M.A.; Kelley, M.; Ginsburg, G.S.; et al. A genomic strategy to refine prognosis in early-stage non-small-cell lung cancer. N. Engl. J. Med. 2006, 355, 570-580. [CrossRef] [PubMed]

11. Steinfort, D.P.; Liew, D.; Conron, M.; Hutchinson, A.F.; Irving, L.B. Cost-benefit of minimally invasive staging of non-small cell lung cancer: A decision tree sensitivity analysis. J. Thorac. Oncol. 2010, 5, 1564-1570. [CrossRef] [PubMed] 
12. Franz, A.M.; Haidegger, T.; Birkfellner, W.; Cleary, K.; Peters, T.M.; Maier-Hein, L. Electromagnetic tracking in medicine-A review of technology, validation, and applications. IEEE Trans. Med. Imaging 2014, 33, 1702-1725. [CrossRef] [PubMed]

13. Wood, B.J.; Zhang, H.; Durrani, A.; Glossop, N.; Ranjan, S.; Lindisch, D.; Levy, E.; Banovac, F.; Borgert, J.; Krueger, S.; et al. Navigation with electromagnetic tracking for interventional radiology procedures: A feasibility study. J. Vasc. Interv. Radiol. 2005, 16, 493-505. [CrossRef] [PubMed]

14. Chang, J.; Suh, T.S.; Lee, D.S. Development of a deformable lung phantom for the evaluation of deformable registration. J. Appl. Clin. Med. Phys. 2010, 11, 281-286. [CrossRef]

15. Kairn, T.; Crowe, S.B.; Markwell, T. Use of 3D printed materials as tissue-equivalent phantoms. In IFMBE Proceedings, Proceedings of the World Congress on Medical Physics and Biomedical Engineering, Toronto, Canada, 7-12 June 2015; Jaffray, D.A., Ed.; Springer: Cham, Switzerland, 2015; Volume 51, pp. 728-731.

16. Followill, D.S.; Evans, D.R.; Cherry, C.; Molineu, A.; Fisher, G.; Hanson, W.F.; Ibbott, G.S. Design, development, and implementation of the radiological physics center's pelvis and thorax anthropomorphic quality assurance phantoms. Med. Phys. 2007, 34, 2070-2076. [CrossRef] [PubMed]

17. Bui, J.T.; Gaba, R.C.; Knuttinen, M.G.; Omene, B.O.; Shon, A.; Martinez, B.K.; Owens, C.A. Microwave lung ablation complicated by bronchocutaneous fistula: Case report and literature review. In Seminars in Interventional Radiology; Thieme Medical Publishers: Stuttgart, Germany, 2011; Volume 28, p. 152.

18. Carrafiello, G.; Mangini, M.; Fontana, F.; Di Massa, A.; Ierardi, A.M.; Cotta, E.; Piacentino, F.; Cardim, L.N.; Pellegrino, C.; Fugazzola, C. Complications of microwave and radiofrequency lung ablation: Personal experience and review of the literature. Radiol. Med. 2012, 117, 201-213. [CrossRef] [PubMed]

19. Townsend, C.M.; Beauchamp, R.D.; Evers, B.M.; Mattox, K.L. Textbook of Surgery, 19th ed.; Elsevier: Philadelphia, PA, USA, 2012; pp. 1182-1226.

20. Mikhail, A.S.; Negussie, A.H.; Graham, C.; Mathew, M.; Wood, B.J.; Partanen, A. Evaluation of a tissue-mimicking thermochromic phantom for radiofrequency ablation. Med. Phys. 2016, 43, 4304. [CrossRef] [PubMed]

21. Zhou, Y.; Thiruvalluvan, K.; Krzeminski, L.; Moore, W.H.; Xu, Z.; Liang, Z. CT-guided robotic needle biopsy of lung nodules with respiratory motion-experimental system and preliminary test. Int. J. Med. Robot. Comput. Assist. Surg. 2013, 9, 317-330. [CrossRef] [PubMed] 